\title{
On the LQG theory with bounded control
}

\author{
D. V. Iourtchenko, J. L. Menaldi and A. S. Bratus
}

\begin{abstract}
We consider a stochastic optimal control problem in the whole space, where the corresponding HJB equation is degenerate, with a quadratic running cost and coefficients with a linear growth. In this paper we provide full mathematical details on the key estimate relating the asymptotic behavior of the solution as the space variables tend to infinite.
\end{abstract}

Mathematics Subject Classification (2000). Primary 93E20, Secondary 49J15.

Keywords. Optimal control, Stochastic control, Hamilton-Jacobi-Bellman equation, Asymptotic behavior.

\section{Introduction}

In the previous paper [2] a problem of stochastic optimal control of a dynamic system was considered. Such problems arise in different fields of engineering, namely mechanical, electrical, thermal and others. An equation of motion of this type of systems usually is governed by a stochastic differential equation (SDE) of second or higher order. Written in a state-space form this SDE is transformed into a set of first order SDEs, with noise entering only some of them. As a result the covariance matrix will be degenerate, which may serve as a characteristic feature of this type of systems.

The dynamic programming approach (DPA) may be used to study the problem of optimal control $[4,5]$. It converts the problem of finding an optimal control policy to a problem of finding a solution to degenerate, multidimensional parabolic PDE - Hamilton-Jacobi-Bellman (HJB) equation. The major difficulty here is that the stated Cauchy problem for the HJB equation should be solved in the entire state-space, whereas it cannot be solved numerically, since the asymptotic behavior of the Bellman function is unknown. There is also no general approach to find an analytical solution to the nonlinear, multidimensional, degenerate parabolic PDE. To overcome this complication the hybrid solution method has been proposed. This method suggests finding an analytical function, which satisfies the HJB equation and the initial conditions 
within a certain "outer" domain. This function can be used as a boundary condition for numerical simulation of the corresponding HJB equation, thereby solving the HJB equation within the entire state-space domain. On the other hand, because no boundary conditions are used on the "outer" domain, this degenerated parabolic PDE cannot have a unique solution. However, if we prove that the constructed function provides an asymptotic behavior of the Bellman function, then it can be used as a boundary condition for numerical simulation of the HJB equation. This approach with tedious proof has been given in [2]. In this paper we propose a much shorter proof, which can easily be extended to a dynamic system with multiple-degrees-of-freedom, to dynamic systems under Poisson noise, nonautonomous systems and even deterministic system.

\section{Problem statement}

Consider a dynamical system governed by the following set of SDE:

$$
\left\{\begin{array}{l}
\dot{x_{1}}(s)=x_{2}(s) \\
\dot{x_{2}}(s)=-2 \alpha x_{2}(s)-\beta^{2} x_{1}(s)+v(s)+\sigma \dot{B}(s), \quad t<s \leq T, \\
x(t)=x_{0}, \dot{x}(t)=\dot{x}_{0}
\end{array}\right.
$$

Here $\alpha, \beta, \sigma$ are positive constants, $B=B(s)$ is a Wiener process, derivative of which should be understood formally and the control $v=v(s)$ is an adapted random process satisfying $|v(s)| \leq R$, for a fixed constant $R>0$. The control goal is to minimize the quadratic cost function:

$$
J_{x_{1}, x_{2}, t}(v)=E\left\{\frac{a}{2}\left[\beta^{2} x_{1}^{2}(T)+x_{2}^{2}(T)\right]+\int_{t}^{T} \frac{b}{2}\left[\beta^{2} x_{1}^{2}(s)+x_{2}^{2}(s)\right] \mathrm{d} s\right\}
$$

where $T$ is given constant. A special case of $a=1 / 2$ or $b=1 / 2$ corresponds to the minimization of the mean total system response energy. Following the DPA to solve the problem (1.1), (1.2) we introduce the Bellman function

$$
u\left(x_{1}, x_{2}, t\right)=\inf \left\{J_{x_{1}, x_{2}, t}(v):|v(\cdot)| \leq R\right\},
$$

which satisfies the following Hamilton-Jacobi-Bellman equation

$$
\begin{aligned}
& \frac{\partial u}{\partial t}+L u+\inf _{|v| \leq R}\left\{v \frac{\partial u}{\partial x_{2}}\right\}+F=0, \\
& F\left(x_{1}, x_{2}\right)=b / 2\left(\beta^{2} x_{1}^{2}+x_{2}^{2}\right), \\
& L u=x_{2} \frac{\partial u}{\partial x_{1}}-\left(2 \alpha x_{2}+\beta^{2} x_{1}\right) \frac{\partial u}{\partial x_{2}}+\frac{\sigma^{2}}{2} \frac{\partial^{2} u}{\partial x_{2}^{2}} .
\end{aligned}
$$

Equation (1.4) is degenerate parabolic equation, to be solved in the entire state-space with the terminal condition

$$
u\left(x_{1}, x_{2}, T\right)=\frac{a}{2}\left(\beta^{2} x_{1}^{2}+x_{2}^{2}\right) .
$$


Note that

$$
\inf _{|v| \leq R}\left\{v \frac{\partial u}{\partial x_{2}}\right\}=-R\left|\frac{\partial u}{\partial x_{2}}\right| .
$$

It should be mentioned that the stated problem slightly differs from the standard LQG, since the control is not included into the cost function (1.2). However, it can be seen that the proposed strategy allows one to include the term $Q v^{2}$, where $Q$ is a nonnegative constant, into the cost function. Then one would have to look for infinum of $\inf _{|v| \leq R}\left(Q v^{2}+v \partial_{2} u\right)$. Since $Q>0$ then we have a parabola with its minimum at $\partial_{2} u / 2 Q$. If $\left|\partial_{2} u / 2 Q\right|<R$ then the minimum will be at $\partial_{2} u / 2 Q$, which means that in terms of control policy we have the standard LQG problem. If $\left|\partial_{2} u / 2 Q\right|>R$ then the minimum is at $v=R$ for $\partial_{2} u<0$, and $v=-R$ for $\partial_{2} u>0$, which means $v=-R \operatorname{sign}\left(\partial_{2} u\right)$. Thus we get the control policy similar to one with no control force entering the cost function.

\section{Construction of a "solution" and the first order estimate}

It is possible to construct a function, which would satisfy HJB equation (1.4) and initial condition (1.5) within a certain "outer" domain. Since this function does not satisfies the HJB equation in the entire state-space, we cannot call it the solution. To express the following arguments as clear as possible consider, for the time being, the case of $\alpha=0$ (more general case can be treated with minor modifications).

To construct the solution within the "outer" domain assume the existence of a domain $\Gamma\left(x_{1}, x_{2}, t\right)$, which does not contain a switching line $\left(\partial u / \partial x_{2}=0\right)$ for any $x_{1}, x_{2}$ and $t>0$, see [2] for details. Thus, within this domain $z=$ $\operatorname{sign}\left(\partial u / \partial x_{2}\right)$ is a constant. Then looking for a solution in the form $f_{i j}(t) x_{i} x_{j}$ $\left(x_{0}=1\right)$ results in a set of ODEs for $f_{i j}(t)$ with appropriate initial conditions [6]. Solving analytically these ODEs one derives the "solution" to HJB equation (1.4) (with $b=0$ for simplicity) as

$$
\begin{aligned}
\tilde{u}\left(x_{1}, x_{2}, \tau\right)= & \frac{a}{2}\left(\left[x_{2}-\frac{R z}{\beta} \sin (\beta[T-t])\right]^{2}\right. \\
& \left.+\left[\beta x_{1}+\frac{R z}{\beta}(1-\cos (\beta[T-t]))\right]^{2}\right)+\frac{\sigma^{2}(T-t)}{2} a .
\end{aligned}
$$

Note that the last term in the equation appears from integrating the noise intensity with respect to time, so if $\sigma=\sigma(\tau)$ then the last term would be $1 / 2 \int_{0}^{\tau} \sigma^{2}(s) \mathrm{d} s$. This function $\tilde{u}$ satisfies the HJB equation (1.4) and initial conditions (1.5) within the following "outer" domain

$$
\left|x_{2}\right| \geq \frac{R}{\beta}|\sin \beta(T-t)| .
$$

A substitution shows that $\tilde{u}$ defined by (2.1) satisfies the HJB equation (1.4) within the "outer" domain given by the condition (2.2). Once the analytical "solution" is known, it can be used as a boundary condition to solve the HJB 
equation numerically within the remaining "inner" domain, thereby finding a solution to the corresponding HJB equation in the entire state-space. It is worth mentioning that, since the system (1.1) does not depend on time explicitly, a "solution" for the case of $b \neq 0$, can be obtained by integrating (2.1) with respect to explicit time $\tau$.

In order to derive the first approximation, let's consider the quadratic part of the function (2.1) and its integral only ( $a \geq 0, b \geq 0$ are constants)

$$
\Psi\left(x_{1}, x_{2}, t\right)=\frac{1}{2}\left(\beta^{2} x_{1}^{2}+x_{2}^{2}\right)[a+b(T-t)],
$$

which satisfies $L \Psi=\frac{\sigma^{2}}{2}[a+b(T-t)]$. Define $w\left(x_{1}, x_{2}, t\right)=u\left(x_{1}, x_{2}, t\right)-$ $\Psi\left(x_{1}, x_{2}, t\right)$, where $u\left(x_{1}, x_{2}, t\right)$ is the solution of HJB equation (1.4) with $\alpha=0$. Then in the paper [2] the authors have obtained the following estimate

$$
\lim _{\left|x_{1}\right|+\left|x_{2}\right| \rightarrow \infty} \frac{\left|u\left(x_{1}, x_{2}, t\right)-\Psi\left(x_{1}, x_{2}, t\right)\right|}{\left|x_{1}\right|^{2}+\left|x_{2}\right|^{2}}=0,
$$

Note, that the equality (2.4) holds in the case of poisson noise and the system with linear friction $(\alpha \neq 0)$.

\section{Complete estimate}

A priori, the expression (2.1) defines the function $\tilde{u}$ valid only within the "outer" domain, so that one needs a suitable extension to the remaining "inner" domain. To accomplish this task, we keep almost the same expression, but we replace the function $z=$ sign with a suitable smooth and bounded function $\tilde{z}=\tilde{z}(x, t)$ such that:

$$
\tilde{z}\left(x_{1}, x_{2}, t\right)=\operatorname{sign}\left(x_{2}\right) \text { if }\left|x_{2}\right| \geq R_{0},
$$

with $R_{0}$ satisfying $R_{0} \geq R / \beta$, and some other conditions to be determined below. Recall that $R$ and $\beta$ are the initial constants of the model, and $R / \beta$ represents the size of the "inner" domain.

For the sake of simplicity, in this section we use the local notation $x=$ $\left(x_{1}, x_{2}\right)$ and $a=1, b=0$. If $u$ is the optimal cost and $w=u-\psi$, where $\psi$ is given by (2.1), then we obtain the following nonlinear equation $(\tau=T-t)$

$$
\partial_{\tau} w=L w+\inf _{|v| \leq R}\left\{v\left[\varphi_{2}+\partial_{2} w\right]\right\}+\varphi
$$

where $\varphi_{2}=\left(x_{2}-\frac{R}{\beta} \tilde{z} \sin \beta \tau\right)\left(1-\frac{R}{\beta} \partial_{2} \tilde{z} \sin \beta \tau\right), \varphi=\left(x_{2} R \tilde{z}+\frac{R^{2}}{\beta} \tilde{z}^{2} \sin \beta \tau\right)+\varphi_{1}$, and

$$
\begin{aligned}
\varphi_{1}= & {\left[\left(x_{1} x_{2} R+2 x_{2} \frac{R^{2}}{\beta^{2}} \tilde{z}\right)(1-\cos \beta \tau)-x_{2}^{2} \frac{R}{\beta} \sin \beta \tau\right] \partial_{1} \tilde{z} } \\
& +\left[\left(x_{1} x_{2} \beta-\frac{\sigma^{2}}{\beta}\right) R \sin \beta \tau+R x_{1}\left(x_{1} \beta^{2}+2 \tilde{z} R\right)(\cos \beta \tau-1)\right] \partial_{2} \tilde{z}
\end{aligned}
$$




$$
\begin{aligned}
& +\left(\sigma^{2} \frac{R^{2}}{\beta^{2}}\right)(1-\cos \beta \tau)\left[\left(\partial_{2} \tilde{z}\right)^{2}+\tilde{z} \partial_{2}^{2} \tilde{z}\right] \\
& +\left(\sigma^{2} \frac{R}{2 \beta}\right)\left[x_{1} \beta(1-\cos \beta \tau)-x_{2} \sin \beta \tau\right]\left(\partial_{2}^{2} \tilde{z}\right) .
\end{aligned}
$$

Recall that although we have $\tilde{z}=\tilde{z}(x, t)$, but only the dependency in $x_{2}$ is relevant. In view of (3.1), on the region where $\left|x_{2}\right| \geq R_{0}$ we have $\tilde{z}(x, t)=\operatorname{sign}\left(x_{2}\right)$ and therefore $\partial_{1} \tilde{z}=\partial_{2} \tilde{z}=0$ and $\varphi_{1}=0$. Moreover, if there are constants $C_{1}, k>0$ such that

$$
\left|\partial_{1} \tilde{z}\right|+\left|\partial_{2} \tilde{z}\right|+\sigma^{2}\left|\partial_{2}^{2} \tilde{z}\right| \leq C_{1}\left(1+\left|x_{1}\right|\right)^{-k-1}, \quad \forall x_{1}, x_{2}, t
$$

then for another constant $C>0$ we have $\left|\varphi_{1}\right| \leq C\left(1+\left|x_{1}\right|+\left|x_{2}\right|\right)^{-k}$, for every $x_{1}, x_{2}$, and $t$. Note that if $\sigma=0$ then the second derivative $\partial_{2}^{2} \tilde{z}$ does not intervene, and calculations are simpler.

The expression (3.2) is the Hamilton-Jacobi-Bellman equation corresponding to the following optimal control problem:

$$
\tilde{J}_{x, t}(v)=\mathbb{E}\left\{\int_{t}^{T}\left[v(s) \varphi_{2}(x(s), s)+\varphi(x(s), s)\right] \mathrm{d} s\right\}
$$

and

$$
w(x, t)=\inf _{|v(\cdot)| \leq R}\left\{\tilde{J}_{x, t}(v)\right\} .
$$

This optimal cost $w$, which is equals to $u-\psi$, has to be estimated in the "outer" domain to deduce the desired result.

The running cost $\ell(x, t, v)=v \varphi_{2}(x, t)+\varphi(x, t)$ can be written as $\ell(x, t, v)=\ell_{1}(x, t, v)+\ell_{0}(x, t, v)+\varphi_{1}(x, t)$ with

$$
\begin{aligned}
\ell_{1}(x, t, v)= & {\left[v\left(1-\frac{R}{\beta} \partial_{2} \tilde{z}(x, t) \sin \beta \tau\right)+R \tilde{z}(x, t)\right] x_{2}+} \\
& +v \frac{R^{2}}{\beta^{2}} \tilde{z}(x, t) \partial_{2} \tilde{z}(x, t) \sin ^{2} \beta \tau \\
\ell_{0}(x, t, v)= & \left(\frac{R}{\beta} \tilde{z}(x, t) \sin \beta \tau\right)(-v-R \tilde{z}(x, t)) .
\end{aligned}
$$

Notice that if $\tilde{z}=\operatorname{sign}\left(x_{2}\right)$ then $\ell_{1}=(v+R) x_{2}$ and $\ell_{0}=-\frac{R}{\beta} \sin \beta \tau(v+R)$, so $x_{2}-\frac{R}{\beta} \sin \beta \tau$ determines the sign of $\ell=\ell_{1}+\ell_{0}$, since $\varphi_{1}=0$. Thus, if $\tilde{z}$ and $\partial_{2} \tilde{z}$ are bounded then $\ell_{0}$ is a bounded function, and moreover, within the region $\left|x_{2}\right| \leq R_{0}$ we can bound the function $\ell_{1}$, i.e., $\left|\ell_{1}\right| \leq C\left(1+\left|x_{1}\right|\right)^{-k}$ for any $x, t$ and some constant $C$. Similarly, for $\left|x_{2}\right| \geq R_{0}$, we have two possibilities: (1) $x_{2}>0$ which implies $\tilde{z}=1$ and $\partial_{2} \tilde{z}=0$, and we deduce $\ell_{1}=(v+R) x_{2} \geq 0$, for every $|v| \leq R$; and (2) $x_{2}<0$ which implies $\tilde{z}=-1$ and $\partial_{2} \tilde{z}=0$, and we deduce $\ell_{1}=(v-R) x_{2} \geq 0$, for every $|v| \leq R$. This proves that under the 
conditions (3.1) and (3.3) there exist constants $C_{1}, C_{0}>0$ such that

$$
\begin{aligned}
& \ell_{1}(x, t, v) \geq 0, \quad \forall x, t, v, \text { with }\left|x_{2}\right| \geq R_{0}, \\
& \ell_{1}(x, t, v) \geq-C_{1}\left(1+\left|x_{1}\right|+\left|x_{2}\right|\right)^{-k}, \quad \forall x, t, v, \\
& \ell_{0}(x, t, v) \geq-C_{0}, \quad \forall x, t, v,
\end{aligned}
$$

Thus, we deduce the estimate

$$
\tilde{J}_{x, t}(v) \geq-C\left(1+\left|x_{2}\right|\right)^{-k}(T-t), \quad \forall x, t,
$$

for some suitable constant $C>0$ depending only on the parameters (e.g., $R$ ). Actually, since for every $m>0$ there exists a constant $C_{m}>0$ such that $\ell_{1}(x, t, v) \geq-C_{m}\left(1+\left|x_{1}\right|+\left|x_{2}\right|^{m}\right)^{-k}$, for every $x, t, v$, we deduce that (3.5) holds for $\left|x_{2}\right|$ replaced with $\left|x_{2}\right|^{m}$. Moreover, if we assume that for $v^{*}(x)=$ $-R \operatorname{sign}\left(x_{2}\right)$ we have

$$
\ell\left(x, t, v^{*}(x)\right) \geq-C\left(1+\left|x_{1}\right|\right)^{-k}, \quad \forall x, t, v,
$$

then for every $m>0$ there exists a constant $C_{m}>0$ such that

$$
\tilde{J}_{x, t}\left(v^{*}\right) \geq-C_{m}\left(1+\left|x_{1}\right|+\left|x_{2}\right|^{m}\right)^{-k}(T-t), \quad \forall x, t,
$$

where $v^{*}(t)=v^{*}(x(t))$ is assumed to be an optimal feedback for the initial control problem. Note that if $\left|x_{2}\right| \geq R_{0}$ then $\tilde{z}(x, t)=\operatorname{sign}\left(x_{2}\right)$ and therefore $\ell_{0}\left(x, t, v^{*}(x)\right)=0$, which means that condition (3.6) is only required when $\left|x_{2}\right|<R_{0}$. For instance, if we assume that $|\tilde{z}| \leq 1, \operatorname{sign}(\tilde{z})=\operatorname{sign}\left(x_{2}\right)$ and $\sin \beta \tau \geq 0$ then we deduce $\ell_{0}\left(x, t, v^{*}(x)\right) \geq 0$.

On the other hand, to get a bound from above, we use the smooth function $\tilde{z}$ to define the feedback $v_{0}(t)=-R \tilde{z}(x(t), t)$, which produce a running cost

$$
\begin{aligned}
\ell\left(x, t, v_{0}\right)= & x_{2} \frac{R^{2}}{\beta} \tilde{z}(x, t) \partial_{2} \tilde{z}(x, t) \sin \beta \tau \\
& -\frac{R^{3}}{\beta^{2}} \tilde{z}^{2}(x, t) \partial_{2} \tilde{z}(x, t) \sin ^{2} \beta \tau+\varphi_{1}(x, t),
\end{aligned}
$$

which has the property that $\left|\ell_{0}\left(x, t, v_{0}(t)\right)\right| \leq C\left(1+\left|x_{1}\right|\right)^{-k}$, for every $x, t$ and some suitable constant $C>0$, and also, $\ell\left(x, t, v_{0}\right)=0$ if $\left|x_{2}\right| \geq R_{0}$. This shows that for every $m>0$ there exists a constant $C_{m}>0$, which depend on the data and $m$, such that

$$
\tilde{J}_{x, t}\left(v_{0}\right) \leq C_{m}\left(1+\left|x_{1}\right|+\left|x_{2}\right|^{m}\right)^{-k}(T-t), \quad \forall x, t .
$$

We have proven the following

Theorem 3.1. Let $u$ be the optimal cost of our initial control problem. Then for every $m>0$ there exists a constant $C_{m}>0$ depending only on the data such that

$$
u(x, t)-\psi(x, t) \leq C_{m}\left(1+\left|x_{1}\right|+\left|x_{2}\right|^{m}\right)^{-k}(T-t), \quad \forall x, t,
$$

where $\psi$ is defined by (2.1) with some smooth and bounded function $\tilde{z}$ satisfying (3.1) and (3.3). Moreover, if we accept that $v^{*}(x)=-R \operatorname{sign}\left(x_{2}\right)$ is an optimal feedback for the initial control problem then 


$$
u(x, t)-\psi(x, t) \geq-C_{m}\left(1+\left|x_{1}\right|+\left|x_{2}\right|^{m}\right)^{-k}(T-t), \quad \forall x, t,
$$

provided (3.6) is satisfied. Otherwise, we only have

$$
u(x, t)-\psi(x, t) \geq-C_{m}\left(1+\left|x_{2}\right|\right)^{-m}(T-t), \quad \forall x, t,
$$

for some suitable constant $C_{m}$.

Notice that we use the fact that a solution (with the regularities of $u$ ) of the HJB equation (3.2) is indeed the optimal cost (3.4). For instance, we know that $u$ is the maximum sub-solution (or the unique continuous viscosity solution) $[1,3]$ of the corresponding HJB equation, and because $\psi$ is smooth, $w=u-\psi$ is the maximum sub-solution of the HJB equation (3.2), and therefore, the optimal cost.

To actually see that $v^{*}=-R \operatorname{sign}\left(x_{2}\right)$ is an optimal feedback for the control problem we need to establish the existence of week solution of nondegenerate stochastic differential equations with measurable coefficients, in our case the measurable coefficient is due to the discontinuous feedback. For instance, if we write state equation (1.1) in Itô form with a Borel feedback $v(s)=v\left(x_{1}(s), x_{2}(s)\right)$ then we may assume $x_{1}(\cdot)$ given and solve the stochastic equation in the variable $x_{2}(\cdot)$, using Krylov's results [7], and then integrate to get $x_{1}(\cdot)$. This gives an iterative procedure leading to a weak solution (however, the actual proves is perhaps beyond the scope of this paper). Alternatively, we can show that a weak solution (for any measurable feedback) exists, following an explicit probabilistic construction similar to the solution of the typical one dimensional example $\mathrm{d} x=\operatorname{sign}(x) \mathrm{d} w(t)$. Actually, for our purpose, an argument with $\varepsilon$-optimal controls could be sufficient.

Finally, to construct a function $\tilde{z}$ satisfying the above condition, we may take the function $\operatorname{sign}\left(x_{2}\right)$ for $\left|x_{2}\right| \geq R_{0}$ and make an odd $C^{2}$ extension for $\left|x_{2}\right| \leq R_{0}$, satisfying $|\tilde{z}| \leq 1$ and $\operatorname{sign}(\tilde{z})=\operatorname{sign}\left(x_{2}\right)$.

\section{Conclusions}

Given mathematical proof resulted in the Theorem 3.1 shows that a specially constructed solution provides asymptotic behavior of the Bellman function as the space variable goes to infinite. Consequently, this solution may be used as boundary conditions to solve the HJB equation numerically within a bounded domain. It can be done despite the fact that the inner domain is unbounded in $x_{1}$ since the proven Theorem 3.1 provides estimate for Bellman function in both directions. That means that the proposed earlier hybrid solution method [6] of finding the solution to the stochastic LQ problem of optimal control is a completely formulated and valid new technique. It is important to stress that Theorem 3.1 applies also for for deterministic optimal control LQ problem with bounded control. 


\section{Acknowledgment}

The first author was support by the Council of the Russian Federation President (MD-6147.2008.1) for young scientists. This support is most highly appreciated.

\section{References}

[1] Bardi, M., Capuzzo-Dolcetta, I.: Optimal control and viscosity solutions of Hamilton-Jacobi-Bellman equations. Birkhäuser (1997)

[2] Bratus, A.S., Iourtchenko, D.V., Menaldi, J.-L.: Local solutions to the Hamilton-Jacobi-Bellman equation in stochastic problems of optimal control. Dokl. Math. 74(1), 610-613 (2006)

[3] Crandall, M.G., Lions, P.L.: Viscosity solutions of Hamilton-Jacobi equations. Trans. Am. Math. Soc. 277, 1-42 (1984)

[4] Dreyfus, S.E.: Dynamic Programming and Calculus of Variations. Academic Press, New York (1965)

[5] Fleming, W.H., Soner, H.M.: Controlled Markov Processes and Viscosity Solutions. Springer-Verlag, New York (1992)

[6] Iourtchenko, D.V.: Solution to a class of stochastic LQ problems with bounded control. Automatica 45, 1439-1442 (2009)

[7] Krylov, N.V.: Controlled Diffusion Processes. Springer-Verlag, Berlin (1980)

D. V. Iourtchenko

Department of Mathematical Sciences,

Saint-Petersburg State Polytechnic University,

Saint-Petersburg 195251,

Russia

e-mail: daniil@phmf.spbstu.edu

J. L. Menaldi

Department of Mathematics,

Wayne State University,

Detroit, MI 48202, USA

e-mail: menaldi@wayne.edu

A. S. Bratus

MIIT, Department of Mathematics,

Moscow 127994, Russia

e-mail: bratus@miit.ru

Received: 29 January 2009.

Accepted: 02 March 2010. 